TITLE:

RECENT FORAMINIFERA

THANATOCOENOSES OF BEACH

AND NEARSHORE SEDIMENTS

ALONG THE COAST OF WAKAYAMAKEN, JAPAN

\author{
$\operatorname{AUTHOR}(\mathrm{S})$ : \\ Uchio, Takayasu
}

CITATION:

Uchio, Takayasu. RECENT FORAMINIFERA THANATOCOENOSES OF BEACH AND

NEARSHORE SEDIMENTS ALONG THE COAST OF WAKAYAMA-KEN, JAPAN. PUBLICATIONS

OF THE SETO MARINE BIOLOGICAL LABORATORY 1962, 10(1): 133-144

ISSUE DATE:

1962-07-30

URL:

http://hdl.handle.net/2433/175295

RIGHT: 


\title{
RECENT FORAMINIFERA THANATOCOENOSES OF BEACH AND NEARSHORE SEDIMENTS ALONG THE COAST OF WAKAYAMA-KEN, JAPAN
}

\author{
TAKayasu UCHIO \\ Petroleum Engineering Institute, University of Tokyo
}

With 2 Tables and 1 Text-figure

\section{Introduction}

As a part of the ecological study of Recent Forminifera around the Japanese coast, five beach-sand samples and four nearshore sediment samples along the coast of Wakayama-ken have been studied for a quantitative analysis of Foraminifera assemblages. The locations of these samples are shown in Figure 1.

The beach-sand samples were collected by the writer in April, 1951 at Wakaura, Wakayama-shi ; Shiraki and Nishihiro, Hiro-chô, Arita-gun; Seto (near the Seto Marine Biological Laboratory), Shirahama-chô, Nishimuro-gun; and near the Kushimoto Railway Station, Kushimoto-chô, Nishimuro-gun. Among them, the sample at Kushimoto was collected in a special way as follows. As waves retreat in ebb tide, they leave small patches of white part on the surface of ripple marks. The white patches are, in reality, concentrations of Foraminifera tests by natural processes. As the result, this white part has a much larger population than the other samples per unit weight or volume of sediment, and the characteristics of the Foraminifera assemblage may be different from those collected in an ordinary way. However, the writer believes that the Foraminifera assemblage represents a general aspect of the intertidal assemblage since the sizes of the foraminiferal tests are variable and this fact suggests that the sorting process of sediment particles (including foraminiferal tests) by surf was not too strong there.

The four nearshore sediment samples were collected in and near Mori Harbor by members of the Hydrographic Office of Japan on August 26 and 28, 1954 using a dredge. The exact locations of them are as follows :

Publ. Seto Mar. Biol. Lab., X (1), 1962. (Article 8) 


\begin{tabular}{l|c|c|c|c}
\hline Station No. & 1 & 2 & 3 & 4 \\
\hline Depth (m) & 7.0 & 6.4 & 2.9 & 8.6 \\
N. Lat. & $33^{\circ} 42^{\prime} 48^{\prime \prime}$ & $33^{\circ} 42^{\prime} 57^{\prime \prime}$ & $33^{\circ} 43^{\prime} 00^{\prime \prime}$ & $33^{\circ} 42^{\prime} 37^{\prime \prime}$ \\
E. Long. & $135^{\circ} 23^{\prime} 48^{\prime \prime}$ & $135^{\circ} 23^{\prime} 54^{\prime \prime}$ & $135^{\circ} 24^{\prime} 04^{\prime \prime}$ & $135^{\circ} 23^{\prime} 12^{\prime \prime}$ \\
Sediment & mud & mud & mud & mud \& sand \\
\hline
\end{tabular}

The writer wishes to express his cordial thanks to Mr. Takahiro SAto of the Hydrographic Office of Japan for giving the sediment samples of Mori Harbor to the writer, Prof. Takasi Tokioka of the Seto Marine Biological Laboratory of

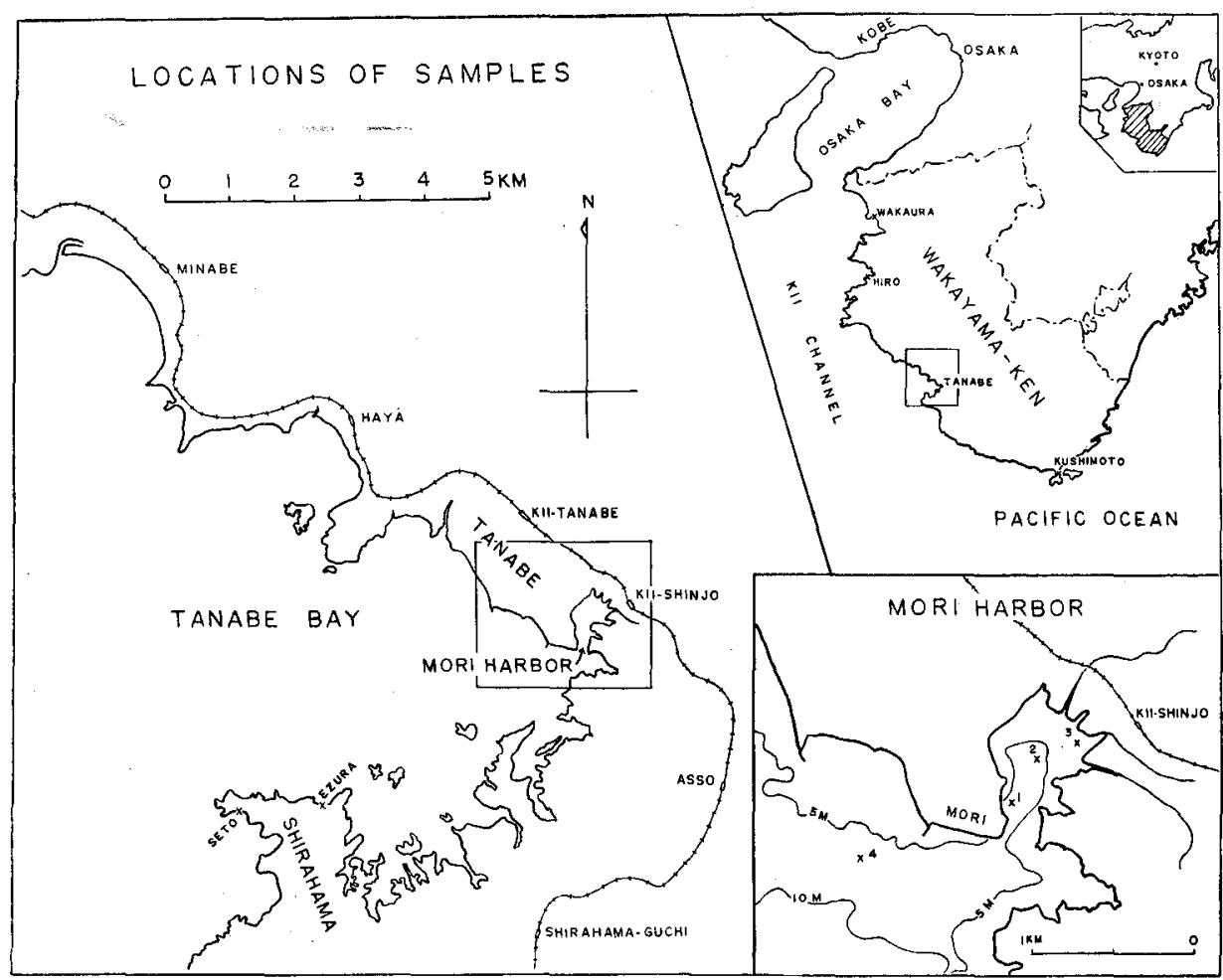

Figure 1.

Kyoto University, and to Prof. Tomofusa Mitsuchi of the writer's institute for their constant encouragements and advices.

\section{General Statements}

The coast under discussion is the western coast of Kii Peninsula. The shoreline is rather complex, showing very many indentations, suggesting recent subsidence of land. The coast is rocky in most part, and the northern part (from 
Wakayama-shi to Minabe-chô, ca. $7 \mathrm{~km}$ northwest of Tanabe-shi) consists of Paleozoic and Mesozoic formations, while the southern part (from Minabe-cho to Kushimoto-chô) consists of Cenozoic (Miocene and Paleogene) formations. Therefore, derived fossil Foraminifera from these formations, if present in the Recent sediment, can be easily distinguished from Recent Foraminifera. The coast is under the influence of the warm Kuroshio Current flowing through Kii Channel towards north.

Concerning the productivity of Tanabe Bay, field surveys were made in November, 1956 and in August, 1957, and fourteen papers were published by Miyadi, Hayami and their collaborators in 1958 and 1959. From these results, the hydrography of Tanabe Bay is briefly described as follows :

(1) November, $1956 \ldots$ “. Water temperature varied from $20.28^{\circ} \mathrm{C}$ to $23.14^{\circ} \mathrm{C}$. Temperatures on the surface layer were more variable than on the other layers. Differences on the surface were $2.76^{\circ} \mathrm{C}$, on both the 5 and 15 meter layers were $1.15^{\circ} \mathrm{C}$, and on the bottom were $1.83^{\circ} \mathrm{C}$. Tracing the isothermal lines, it is recognizable that in general temperatures were lower from the entrance towards the inner parts and warmer waters in the mouth entered towards southeast. As to the vertical distribution, surface temperature was generally higher than bottom temperature and its average range for all stations was $0.4^{\circ} \mathrm{C} \ldots$ The highest chlorinity measured at the surface was $18.96 \%$ and the lowest $14.35 \%$. Open sea water entered the bay in the form of wedge. In the innermost arms and estuaries of the rivers Aizu-gawa and Haya-gawa, discharge of fresh water seemed to have any appreciable effect upon chlorinity of the bay water, showing the low chlorinity less than $18.00 \%$. In Mori arm (harbor), its effect was the most remarkable, but seemed to be confined only to the surface water. Vertical ranges of chlorinity were small, the maximum value being $4.42 \%$ (Mori arm), minimum $0.00 \%$ and mean $0.3 \%$... In general, concentration of inorganic silicate found in the inner region was higher than in the outer, with decreasing tendency towards the open sea. It showed a tendency becoming lower from surface to bottom. Higher concentration was found in the five arms and in the northwest area where the river water pours into the bay $(1.04 \mathrm{mg} / \mathrm{L}$ at the surface water in Mori arm). These facts prove the great effect of fresh water pouring into the bay and coincide well with the distribution figure of surface chlorinity... Inorganic phosphate were distributed in patches. In general, it had a tendency that concentration becomes lower towards the outer region from the innermost and towards the bottom from the surface. High concentrations of phosphate were found on the surface at Mori arm $(0.0487 \mathrm{mg} / \mathrm{L})$, Utinoura $c a .0 .025 \mathrm{mg} / \mathrm{L}$ )..." (Fuse, Yamazi \& Harada, 1958).

(2) August, $1957 \ldots$ The average surface temperature along a traverse at the entrance of the bay ( 5 stations) was $27.8^{\circ} \mathrm{C}$ and the bottom water temperature $23.5^{\circ} \mathrm{C} \ldots$ Along the same traverse, the average surface chlorinity was $18.25 \%$ 
and the average bottom chlorinity was 18.74\% (FUSE, 1959). Chlorinity near the entrance of Mori arm) was $16.88 \%, 18.56 \%$ and $1.75 \%$ at depths of 0.5 and $10 \mathrm{~m}$ respectively (Hayami \& Fukuo, Figs. 4, 5, 6, 1959).

\section{Foraminifera Thanatocoenoses}

Occurrences of the benthonic Foraminifera are listed in Table 1. The high order taxonomic grouping, such as family, is artificial and the recent scheme of Loeblich \& TAppan (1960) is used tentatively in the table. Table 2 is a simplified form of Table 1 to show the general features of the sediment samples. In this table, LOEBLICH \& TAPPAN's Rotaliidae and Discorbidae are grouped in one unit (Cushman's Rotaliidae, 1948), and Buliminidae, Bolivinitidae and Uvigerinidae in one unit (Cushman's Buliminidae).

Grain size analysis of these samples was not made. Percentages of sand (larger than $0.061 \mathrm{~mm}$ in diameter) content of the samples are listed in Tables 1 and 2. In Mori Harbor the sand content increases from head of the harbor (Sta. 3) to the entrance (Sta. 1) and farther offshore (Sta. 4). This may be due to the fact that the inner part of the harbor is protected from strong wave action of Tanabe Bay. Organic content of the harbor sediments is much higher than that of the beach sand. Average size of the beach sand is fine to medium at Kushimoto and coarse at the other localities.

The following generalization of Foraminifera distribution may be made from Tables 1 and 2 :

(1) Types of the Foraminifere assemblages are:

Wakaura …..... Elphidium (mostly advena and jenseni) - Ammonia beccariiMiliolidae Assemblage

Shiraki...........Ammonia nipponica-Elphidium crispum Assemblage.

Nishihiro.........Elphidium (mostly crispum and craticulatum)-Marginopora hemprichii-Miliolidae Assemblage.

Ezura ${ }^{1}$............. Amphistegina lessonii--Elphidium craticulatum Assemblage.

Seto …........... Amphistegina lessonii Assemblage.

Kushimoto …..Elphidium (mostly crispum and jenseni) -Cibicides refulgens - Ammonia beccarii-Mliolidae Assemblage.

Mori Sta. 4…Ammonia beccarii-Elphidium-Miliolidae Assemblage.

Mori Sta. 1….Ammonia beccarii-Elphidium (mainly subincertum) Assembl.

Mori Sta. 2…Ammonia beccarii-Elphidium (mainly subincertum) Assemblage

Mori Sta. 3…Ammonia beccarii-Arenaceous Foraminifera (mainly Trochammina cf. globigeriniformis) Assemblage.

1) More than 3000 specimens were counted at this locality. Important species are Amphistegina lessonii (65\%), Elphidium craticulatum (24\%), Quinqueloculina spp. (10\%). Marginopora hemprichii and Camerina ammonoides are rare. 
Table 1. Occurrences of benthonic Foraminifera in percent of total (living plus dead) population. ( $\mathrm{R}=$ rare)

\begin{tabular}{|c|c|c|c|c|c|c|c|c|c|}
\hline \multirow[b]{2}{*}{ Stations } & \multicolumn{5}{|c|}{ Beach sand } & \multicolumn{4}{|c|}{ Mori Harbor } \\
\hline & 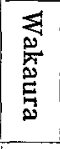 & 总 & 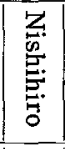 & 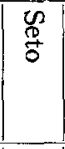 & 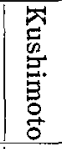 & 3 & 2 & 1 & 4 \\
\hline Depth (m) & 0 & 0 . & 0 & 0 & 0 & 2.9 & 6.4 & 7.0 & 8.6 \\
\hline$\%$ of sand content in sediment & 100 & 100 & 100 & 100 & 100 & 45 & 66 & 65 & 70 \\
\hline Benthonic Population in $5 \mathrm{~g}$ of dried sediment & êे & ठ্心 & $\vec{c}$ & $\sqrt{ \pm}$ & $\begin{array}{l}\underset{\infty}{\infty} \\
\stackrel{\infty}{\infty}\end{array}$ & $\begin{array}{l}\omega \\
\text { S }\end{array}$ & $\underset{\mathbb{I}}{\stackrel{J}{\mid}}$ & 心్స్ & ల్ల \\
\hline $\begin{array}{l}\text { ARENACEOUS FORAMINIFERA } \\
\text { Ammodiscidae } \\
\text { Glomospira gordialis (JONES \& PARKER) }\end{array}$ & - & -1 & - & 一 & - & $\mathbf{R}$ & - & .3 & $\mathbf{R}$ \\
\hline 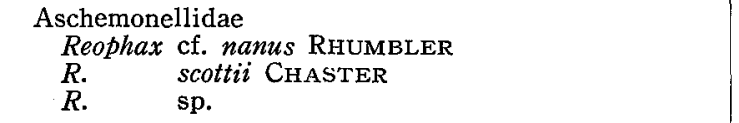 & $\overline{-}$ & Z & $\overline{-}$ & $\bar{z}$ & $\begin{array}{l}- \\
- \\
-\end{array}$ & $\begin{array}{r}1 \\
.9 \\
-\end{array}$ & $\begin{array}{r}.2 \\
.4 \\
-\end{array}$ & $\begin{array}{r}\mathbf{R} \\
\mathrm{R} \\
-\end{array}$ & $\begin{array}{l}\mathrm{R} \\
.2 \\
.2\end{array}$ \\
\hline $\begin{array}{l}\text { Lituolidae } \\
\text { Haplophragmoides sp. } \\
\text { Alveolophragmium cf. jeffreysii (WILLIAMSON) } \\
\text { Ammoscalaria sp. A } \\
\text { Ammobaculites cf. agglutinans (D'ORBIGNY) }\end{array}$ & $\begin{array}{l}- \\
- \\
-\end{array}$ & $\begin{array}{l}- \\
- \\
-\end{array}$ & $\begin{array}{l}- \\
- \\
-\end{array}$ & $\begin{array}{l}- \\
- \\
-\end{array}$ & $\begin{array}{l}- \\
- \\
-\end{array}$ & $\begin{array}{l}1 \\
.6 \\
\mathrm{R}\end{array}$ & $\begin{array}{l}- \\
-1 \\
-\end{array}$ & $\begin{array}{l}- \\
-7 \\
-\end{array}$ & $\begin{array}{l}\overrightarrow{4} \\
2 \\
\mathrm{R}\end{array}$ \\
\hline $\begin{array}{cl}\text { Textulariidae } \\
\text { Textularia } \text { cf. pseudogramen CHAPMAN \& PARR } \\
T . & \text { conica D'ORBIGNY } \\
T . & \text { earlandi PARKER } \\
T . & \text { fistula CUSHMAN } \\
T . & \text { kerimbaensis SAID } \\
T . & \text { sagitula atrata CUSHMAN } \\
T . & \text { cf. candeiana D'ORBIGNY } \\
T . & \text { sp. A } \\
T . & \text { sp. B }\end{array}$ & $\begin{array}{l}\bar{Z} \\
- \\
- \\
- \\
.4 \\
-\end{array}$ & $\begin{array}{l}.8 \\
.6 \\
- \\
-4 \\
.2 \\
- \\
-\end{array}$ & $\begin{array}{l}-7 \\
- \\
- \\
2 \\
- \\
- \\
-\end{array}$ & $\begin{array}{l}- \\
- \\
- \\
- \\
- \\
- \\
-\end{array}$ & $\begin{array}{r}4 \\
2 \\
-2 \\
.2 \\
2 \\
.1 \\
.1 \\
-\end{array}$ & $\begin{array}{l}- \\
- \\
- \\
- \\
- \\
\frac{-}{-3}\end{array}$ & $\begin{array}{l}- \\
-1 \\
- \\
- \\
-\end{array}$ & $\begin{array}{l}- \\
\frac{-}{2} \\
\frac{\mathrm{R}}{-} \\
\frac{\mathrm{R}}{-}\end{array}$ & $\begin{array}{l}-\overline{1} \\
\frac{1}{2} \\
\frac{2}{\mathrm{R}} \\
\frac{-}{-}\end{array}$ \\
\hline $\begin{array}{l}\text { Trochamminidae } \\
\begin{array}{ll}\text { Trochammina } & \text { cf. globigeriniformis (P. \& J.) } \\
T . & \text { cf. pacifica CUSHMAN } \\
T . & \text { squamata PARKER \& JoNES } \\
T . & \text { sp. A } \\
T . & \text { sp. B } \\
\text { Ataxophragmiidae } & \\
\text { Gaudryina cf. niigataensis ASANO }\end{array}\end{array}$ & $\begin{array}{l}- \\
- \\
-\end{array}$ & $\begin{array}{l}- \\
- \\
-\end{array}$ & $\begin{array}{l}- \\
\overline{-} \\
-\end{array}$ & $\begin{array}{l}- \\
- \\
- \\
-\end{array}$ & $\begin{array}{l}- \\
- \\
- \\
-\end{array}$ & $\begin{array}{r}9 \\
-- \\
-6 \\
.6\end{array}$ & $\frac{1}{1}$ & $\begin{array}{c}.8 \\
.1 \\
-1 \\
- \\
-\end{array}$ & $\begin{array}{c}-\overline{6} \\
\mathrm{R} \\
.2 \\
- \\
-\end{array}$ \\
\hline $\begin{array}{l}\text { CALCAREOUS (PORCELAINOUS) FORAMINIFERA } \\
\text { Nubeculariidae } \\
\text { Cornuspira involvens (REUSS) } \\
\text { Vertebralina striata D'ORBIGNY }\end{array}$ & - & $\overline{-}$ & $\overline{.5}$ & - & $\overline{\mathrm{R}}$ & - & - & .3 & $\begin{array}{l}\mathrm{R} \\
\mathrm{R}\end{array}$ \\
\hline $\begin{array}{cl}\text { Miliolidae } & \\
\text { Quinqueloculina agglutinans BRADY (not D'ORBIGNY) } \\
Q . & \text { costata D'ORBIGNY } \\
Q . & \text { cf. elongata NATLAND } \\
Q . & \text { parkeri BRADY } \\
Q . & \text { cf. subarenaria CUSHMAN } \\
Q . & \text { cf. vulgaris D'ORBIGNY } \\
Q . & \text { cf. yabei AsANO } \\
Q . & \text { cf. dainitiensis AsANO } \\
Q . & \text { cf. Sigmoilinopsis schlumbergeri } \\
& \end{array}$ & $\frac{2}{-}$ & $\begin{array}{l}- \\
- \\
- \\
- \\
- \\
- \\
-\end{array}$ & $\begin{array}{l}\frac{.9}{-} \\
\frac{5}{4} \\
\frac{4}{2} \\
-\end{array}$ & $\begin{array}{l}- \\
- \\
.6 \\
.6 \\
6 \\
- \\
- \\
-\end{array}$ & \begin{tabular}{|c}
.2 \\
$\mathrm{R}$ \\
-1 \\
$\mathbf{1}$ \\
2 \\
- \\
2 \\
3 \\
-
\end{tabular} & $\begin{array}{l}\overline{-} \\
\overline{-} \\
\mathrm{R} \\
\overline{-} \\
\overline{-} \\
-\end{array}$ & $\begin{array}{c}- \\
- \\
- \\
\mathrm{R} \\
- \\
- \\
- \\
-\end{array}$ & $\begin{array}{c}\mathrm{R} \\
.4 \\
\mathrm{R} \\
\overline{-} \\
.1 \\
\mathrm{R}\end{array}$ & $\begin{array}{c}- \\
\overline{3} \\
.2 \\
-\bar{R} \\
\overline{\mathrm{R}} \\
3\end{array}$ \\
\hline
\end{tabular}




\begin{tabular}{|c|c|c|c|c|c|c|c|c|c|}
\hline \multirow[b]{2}{*}{ Stations } & \multicolumn{5}{|c|}{ Beach sand } & \multicolumn{4}{|c|}{ Mori Harbor } \\
\hline & 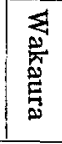 & 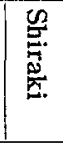 & 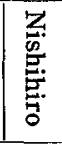 & $\frac{8}{0}$ & 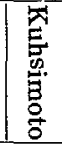 & 3 & 2 & 1 & 4 \\
\hline Depth (m) & 0 & 0 & 0 & 0 & 0 & 2.9 & 6.4 & 7.0 & 8.6 \\
\hline$\%$ of sand content in sediment & 100 & 100 & 100 & 100 & 100 & 45 & 66 & 65 & 70 \\
\hline Benthonic Population in $5 \mathrm{~g}$ of dried sediment & 㞁 & 察 & 忠 & $\underset{\mathbb{A}}{\mathbb{A}}$ & $\begin{array}{c}\mathbb{\infty} \\
\underset{\infty}{\infty} \\
\stackrel{\leftrightarrow}{*}\end{array}$ & 㟒 & 突 & జ్తు & N్ \\
\hline 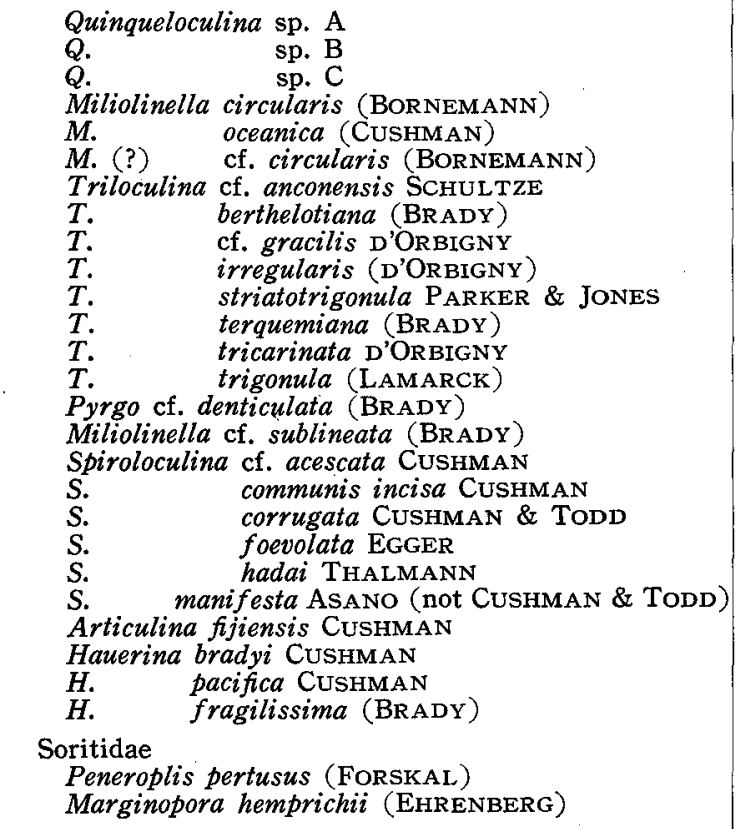 & $\begin{array}{l}- \\
- \\
-8 \\
- \\
- \\
\overline{-} \\
-4 \\
- \\
- \\
\overline{-} \\
- \\
- \\
- \\
- \\
- \\
- \\
- \\
-\end{array}$ & $\begin{array}{l}- \\
- \\
- \\
- \\
- \\
- \\
- \\
- \\
- \\
- \\
- \\
- \\
- \\
- \\
- \\
- \\
- \\
- \\
- \\
-\end{array}$ & $\begin{array}{c}5 \\
-\overline{\mathrm{R}} \\
\mathrm{R} \\
\mathrm{R} \\
.2 \\
.2 \\
- \\
- \\
2 \\
.7 \\
.2 \\
3 \\
- \\
.2 \\
.2 \\
- \\
6 \\
\mathrm{R} \\
-\end{array}$ & $\begin{array}{l}- \\
- \\
- \\
- \\
- \\
- \\
- \\
- \\
- \\
- \\
- \\
- \\
- \\
- \\
- \\
- \\
- \\
- \\
- \\
- \\
-\end{array}$ & $\begin{array}{l}.9 \\
.1 \\
.3 \\
- \\
- \\
- \\
- \\
- \\
- \\
.7 \\
2 \\
3 \\
- \\
.1 \\
.1 \\
\mathrm{R} \\
.1 \\
- \\
3 \\
\mathrm{R}\end{array}$ & $\begin{array}{l}- \\
- \\
- \\
- \\
- \\
- \\
- \\
\overline{\mathrm{R}} \\
- \\
- \\
- \\
- \\
- \\
- \\
\mathrm{R} \\
- \\
- \\
- \\
- \\
-\end{array}$ & $\begin{array}{l}- \\
- \\
- \\
- \\
2 \\
- \\
- \\
- \\
-2 \\
- \\
- \\
- \\
- \\
- \\
- \\
- \\
- \\
-\end{array}$ & 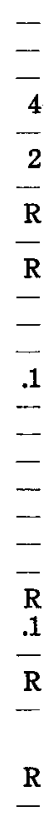 & $\begin{array}{c}- \\
1 \\
4 \\
3 \\
- \\
- \\
\mathrm{R} \\
\mathrm{R} \\
\\
.6 \\
\mathrm{R} \\
.2 \\
.2 \\
\mathrm{R} \\
- \\
.4 \\
.2 \\
\mathrm{R} \\
\end{array}$ \\
\hline $\begin{array}{l}\text { ALCAREOUS (HYALINE) FORAMINIFERA } \\
\text { Nodosariidae } \\
\text { Lagena perlucida (MONTAGU) } \\
\text { L. striata strumosa REUSS } \\
\text { Robulus sp. }\end{array}$ & $\overline{-}$ & $\bar{E}$ & $\overline{-}$ & 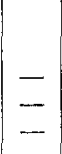 & $\overline{-}$ & - & E & $\begin{array}{l}.1 \\
\mathrm{R} \\
-\end{array}$ & $\begin{array}{l}\mathbf{R} \\
\mathbf{R} \\
-\end{array}$ \\
\hline 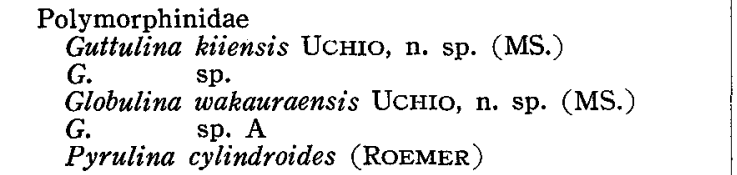 & $\begin{array}{l}\frac{7}{8} \\
-8 \\
.4\end{array}$ & $\begin{array}{l}- \\
- \\
- \\
-\end{array}$ & $\begin{array}{l}- \\
- \\
- \\
-\end{array}$ & $\begin{array}{l}- \\
- \\
- \\
-\end{array}$ & $\frac{\mathbf{R}}{\overline{\mathbf{R}}}$ & $\begin{array}{l}E \\
z \\
-\end{array}$ & $\begin{array}{l}- \\
-\end{array}$ & $\begin{array}{l}- \\
- \\
-\end{array}$ & $\begin{array}{l}\overline{\mathrm{R}} \\
- \\
-\end{array}$ \\
\hline $\begin{array}{l}\text { Glandulinidae } \\
\text { Oolina acuticosta (REUSS) } \\
\text { O. bifurcata.(LEROY) } \\
\text { O. melo D'ORBIGNY } \\
\text { Fissurina cf. cucurbitasema LOEBLICH \& TAPPAN } \\
\text { F. kiiensis UCHIO, n. sp. (MS.) } \\
\text { Turrilinidae } \\
\text { Buliminella elegantissima (D'ORBIGNY) } \\
\text { Buliminoides williamsoniana (BRADY) }\end{array}$ & $\begin{array}{l}E \\
E \\
-\end{array}$ & $\begin{array}{l}- \\
- \\
- \\
-\end{array}$ & $\begin{array}{l}- \\
- \\
- \\
-\end{array}$ & $\begin{array}{l}- \\
- \\
- \\
-\end{array}$ & $\begin{array}{l}\overline{\mathrm{R}} \\
\mathrm{R} \\
\overline{\mathrm{R}}\end{array}$ & $\begin{array}{l}\overline{-} \\
- \\
-\end{array}$ & $\bar{I}=$ & $\begin{array}{l}E \\
E \\
-\end{array}$ & $\begin{array}{l}.2 \\
- \\
.4\end{array}$ \\
\hline
\end{tabular}




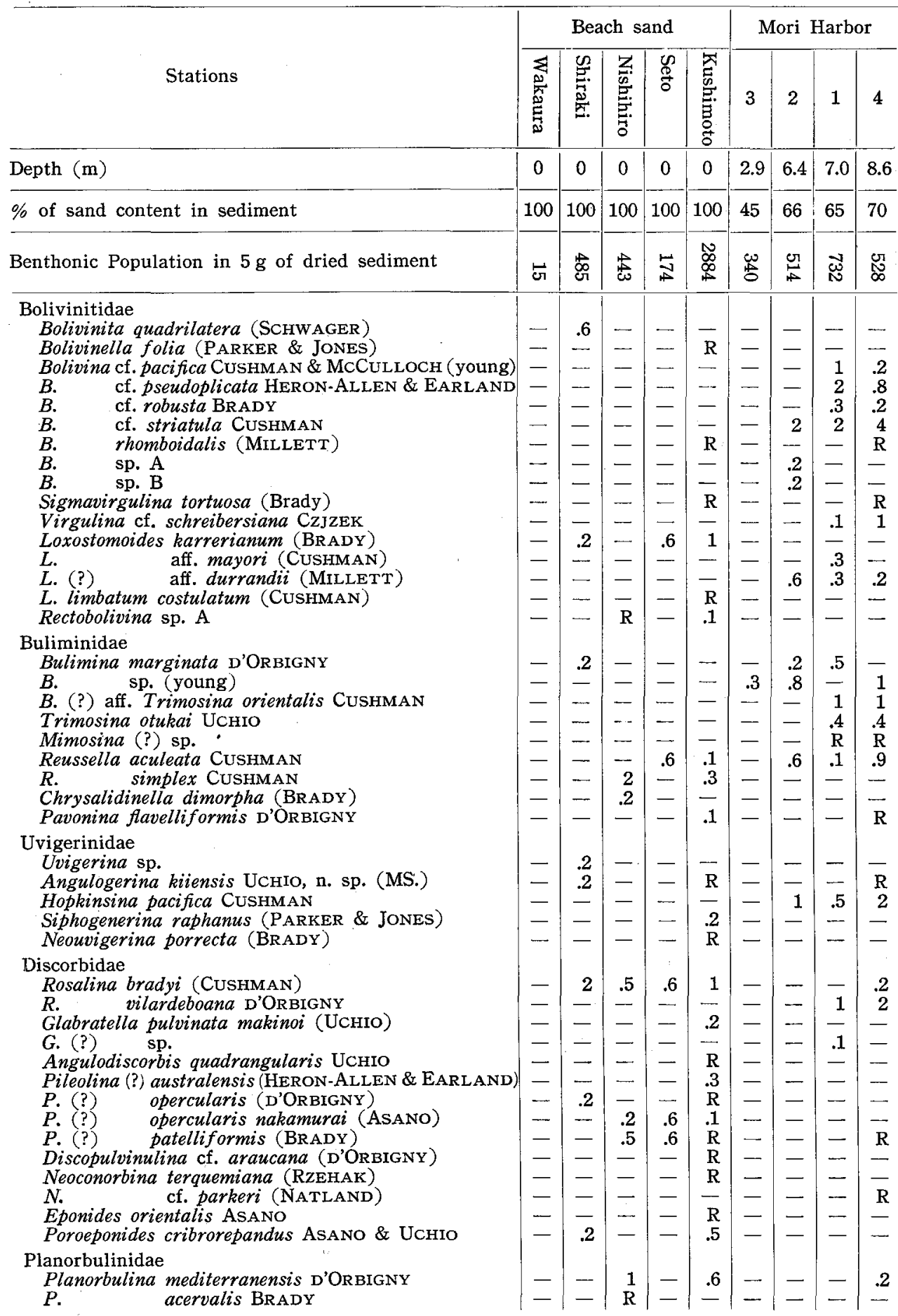




\begin{tabular}{|c|c|c|c|c|c|c|c|c|c|}
\hline \multirow[b]{2}{*}{ Stations } & \multicolumn{5}{|c|}{ Beach sand } & \multicolumn{4}{|c|}{ Mori Harbor } \\
\hline & 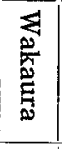 & 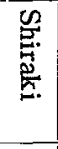 & 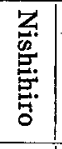 & $\underset{\mathbb{O}}{0}$ & 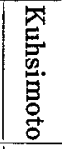 & 3 & 2 & 1 & 4 \\
\hline Depth (m) & 0 & 0 & 0 & 0 & 0 & 2.9 & 6.4 & 7.0 & 8.6 \\
\hline$\%$ of sand content in sediment & 100 & 100 & 100 & 100 & 100 & 45 & 66 & 65 & 70 \\
\hline Benthonic Population in $5 \mathrm{~g}$ of dried sediment & $\overrightarrow{\mathrm{r}}$ & $\stackrel{\infty}{c}$ & 悹 & $\overrightarrow{\mathbf{A}}$ & $\underset{\infty}{\infty}$ & $\begin{array}{l}\omega \\
\text { 岁 }\end{array}$ & 品 & జ్లు & N్ల \\
\hline $\begin{array}{l}\text { Rotaliidae } \\
\text { Ammonia beccarii asanoi UCHIO } \\
\text { A. } \quad \text { beccarii kiiensis UCHIO, n. var. (MS.) } \\
\text { A. } \quad \text { erinacea (HERON-ALLEN \& EARLAND) } \\
\text { A. } \quad \text { aff. inflata (SEGUENZA) } \\
\text { A. nipponica (ASANO) } \\
\text { A. tanabensis UCHIO, n. sp. (MS.) } \\
\text { Pseudorotalia (?) gaimardii (D'ORBIGNY) }\end{array}$ & $\begin{array}{l}28 \\
- \\
- \\
- \\
-\end{array}$ & $\begin{array}{c}.2 \\
-\overrightarrow{3} \\
3 \\
37 \\
.2\end{array}$ & $\begin{array}{c}3 \\
- \\
- \\
- \\
- \\
-\end{array}$ & $\begin{array}{c}- \\
- \\
- \\
2 \\
- \\
-\end{array}$ & $\begin{array}{r}13 \\
2 \\
- \\
- \\
\frac{2}{\mathrm{R}}\end{array}$ & $\begin{array}{c}76 \\
3 \\
- \\
- \\
- \\
-\end{array}$ & $\begin{array}{l}\frac{61}{9} \\
- \\
- \\
-\end{array}$ & $\begin{array}{c}\frac{40}{8} \\
- \\
-\mathrm{R} \\
-\end{array}$ & $\begin{array}{l}\frac{26}{-} \\
-6 \\
- \\
- \\
\end{array}$ \\
\hline 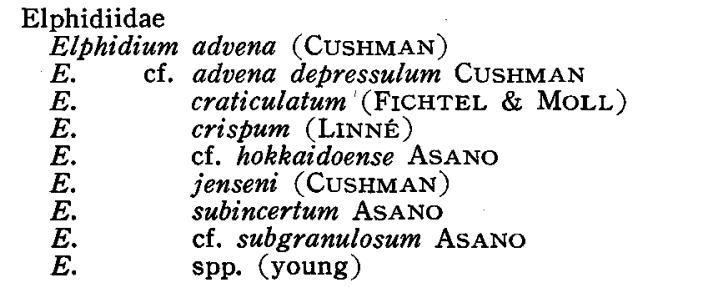 & $\begin{array}{r}15 \\
6 \\
1 \\
.4 \\
1 \\
15 \\
.8 \\
1 \\
-\end{array}$ & $\begin{array}{l}- \\
\overline{-} \\
\overline{31} \\
- \\
- \\
- \\
-\end{array}$ & $\begin{array}{l}-\overline{-} \\
10 \\
19 \\
\frac{2}{2} \\
- \\
-\end{array}$ & $\begin{array}{l}- \\
- \\
6 \\
2 \\
- \\
- \\
- \\
-\end{array}$ & $\begin{array}{r}.2 \\
2 \\
17 \\
\mathrm{R} \\
7 \\
\mathrm{R} \\
- \\
-\end{array}$ & $\begin{array}{r}.9 \\
2 \\
- \\
-9 \\
-9 \\
1 \\
-\end{array}$ & $\begin{array}{r}.2 \\
1 \\
- \\
-2 \\
- \\
7 \\
2 \\
\end{array}$ & $\begin{array}{r}- \\
4 \\
.1 \\
.8 \\
.1 \\
10 \\
1 \\
3\end{array}$ & $\begin{array}{r}-\overline{4} \\
- \\
\overline{2} \\
\mathrm{R} \\
9 \\
2 \\
3\end{array}$ \\
\hline $\begin{array}{l}\text { Nummulitidae } \\
\text { Camerina ammonoides (GRONOVIUS) }\end{array}$ & - & - & - & .6 & -- & $\cdots$ & - & - & - \\
\hline $\begin{array}{l}\text { Anomalinidae } \\
\text { Anomalina glabrata CUSHMAN }\end{array}$ & - & -- & .2 & - & $\mathbf{R}$ & - & - & - & - \\
\hline $\begin{array}{l}\text { Amphisteginidae } \\
\text { Amphistegina lessonii D'ORBIGNY }\end{array}$ & - & - & 4 & 71 & .1 & - & - & - & - \\
\hline $\begin{array}{l}\text { Cymbaloporidae } \\
\text { Cymbaloporetta bradyi (CUSHMAN) }\end{array}$ & - & - & 2 & - & 1 & $=$ & - & - & - \\
\hline $\begin{array}{l}\text { Nonionidae } \\
\text { Nonion cf. granuliferum (TERQUEM) } \\
N . \quad \text { cf. boueanum (D'ORBIGNY) } \\
N . \quad \text { cf. japonicum ASANO } \\
\text { Nonionella stella CUSHMAN \& MOYER } \\
\text { Pseudononion grateloupi (D'ORBIGNY) } \\
P . \quad \text { cf. japonicum (ASANO) } \\
\text { Astrononion cf. gallowayi LOEBLICH \& TAPPAN }\end{array}$ & $\begin{array}{l}- \\
- \\
- \\
- \\
-\end{array}$ & $\begin{array}{l}- \\
- \\
- \\
- \\
-\end{array}$ & $\begin{array}{l}- \\
- \\
- \\
- \\
-\end{array}$ & $\begin{array}{l}- \\
- \\
- \\
- \\
- \\
-\end{array}$ & $\begin{array}{l}\overline{\mathrm{R}} \\
- \\
- \\
\overline{.3} \\
-\end{array}$ & $\begin{array}{l}- \\
- \\
- \\
- \\
-\end{array}$ & $\begin{array}{l}- \\
- \\
- \\
- \\
-\end{array}$ & $\begin{array}{c}\bar{R} \\
.1 \\
\mathrm{R} \\
.3 \\
\mathrm{R}\end{array}$ & $\begin{array}{l}.2 \\
.2 \\
.2 \\
\mathrm{R} \\
\mathrm{R} \\
2 \\
\mathrm{R}\end{array}$ \\
\hline 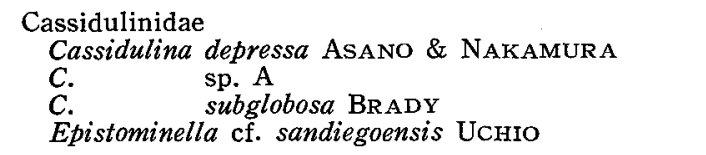 & $\begin{array}{l}- \\
- \\
-\end{array}$ & $\overline{.}$ & $\begin{array}{l}- \\
-\end{array}$ & $\begin{array}{l}- \\
- \\
-\end{array}$ & $\begin{array}{l}\overline{\mathrm{R}} \\
.1 \\
-\end{array}$ & $\begin{array}{l}- \\
-\end{array}$ & $\begin{array}{l}.4 \\
- \\
-\end{array}$ & $\frac{1}{-}$ & $\frac{1}{2}$ \\
\hline $\begin{array}{l}\text { Cibicididae } \\
\text { Cibicides lobatulus (WALKER \& JACOB) } \\
\text { C. cf. pseudoungerianus (CUSHMAN) } \\
\text { C. refulgens (MONTFORT) } \\
\text { Hanzawaia nipponica ASANO }\end{array}$ & $\begin{array}{l}- \\
- \\
-\end{array}$ & $\begin{array}{l}.6 \\
.4 \\
9 \\
2\end{array}$ & $\begin{array}{c}.2 \\
-3 \\
-\end{array}$ & $\begin{array}{c}- \\
- \\
-\end{array}$ & $\begin{array}{r}.2 \\
.6 \\
24 \\
.2\end{array}$ & $\begin{array}{l}- \\
- \\
-\end{array}$ & $\begin{array}{l}\overline{-} \\
.2\end{array}$ & $\begin{array}{l}- \\
-\end{array}$ & $\begin{array}{l}\overline{\mathbf{R}} \\
-\end{array}$ \\
\hline $\begin{array}{l}\text { Spirilinidae } \\
\text { Patellina corrugata WILLIAMSON } \\
\text { Spirillina vivipara EHRENBERG }\end{array}$ & - & $\overline{.2}$ & - & - & - & - & - & .1 & $\overline{-}$ \\
\hline
\end{tabular}


Table 2. Some characteristics of the sediment samples.

\begin{tabular}{|c|c|c|c|c|c|c|c|c|}
\hline & \multicolumn{4}{|c|}{ Beach Sand } & \multicolumn{4}{|c|}{ Mori Harbor } \\
\hline & Wakaura & Nishihiro & Seto & Kushimoto & 3 & 2 & 1 & 4 \\
\hline Depth (m) & 0 & 0 & 0 & 0 & 2.9 & 6.4 & 7.0 & 8.6 \\
\hline Sand Content $(\%)$ & 100 & 100 & 100 & 100 & 45 & 66 & 65 & 70 \\
\hline $\begin{array}{l}\text { Benthonic Foram. Population } \\
\text { in } 5 \mathrm{~g} \text { of dry sediment }\end{array}$ & 15 & 443 & 174 & 2884 & 340 & 514 & 732 & 528 \\
\hline Arenaceous Foram. & $0.4 \%$ & $2 \%$ & $4 \%$ & $9 \%$ & $18 \%$ & $6 \%$ & $5 \%$ & $5 \%$ \\
\hline $\begin{array}{l}\text { Calcareous (Porcelainous) } \\
\text { Foram. }\end{array}$ & 19.2 & 45.3 & 8.4 & 17.6 & $\mathrm{R}$ & 2.2 & 7 & 12.2 \\
\hline $\begin{array}{l}\text { Calcareous (Hyaline) } \\
\text { Foraminifera }\end{array}$ & 80.4 & 52.7 & 87.6 & 73.4 & 82 & 91.8 & 88 & 82.8 \\
\hline Miliolidae & 19 & 32 & 8 & 17 & $\mathrm{R}$ & 2 & 7 & 17 \\
\hline Elphidiidae & 42 & 31 & 8 & 25 & 5 & 12 & 18 & 20 \\
\hline Rotaliidae plus Discorbidae & 28 & 4 & 4 & 19 & 76 & 70 & 49 & 33 \\
\hline $\begin{array}{l}\text { Buliminidae plus Bolivini- } \\
\text { tidae plus Uvigerinidae }\end{array}$ & 0 & 2 & 1 & 2 & 1 & 10 & 16 & 19 \\
\hline Polymorphinidae & 8 & 0 & 0 & 0 & 0 & 0 & 0 & $\mathbf{R}$ \\
\hline Amphisteginidae & 0 & 4 & 71 & 0.1 & 0 & 0 & 0 & 0 \\
\hline Miscellaneous Families & 3 & 21 & 5 & 3 & 0 & 0 & 5 & 0 \\
\hline Tropical Foraminifera & 3 & 48 & 80 & 10 & 0.3 & 11 & 9 & 10 \\
\hline
\end{tabular}

Thus, the beach sand is characterized by high percentages of Elphidium and Miliolidae except at Ezura and Seto where Amphistegina lessonii is predominant. Rotaliidae (mostly Ammonia) and Anomalinidae (mostly Cibicides) are also important in beach sand. Harbour samples are mud and are characterized by predominance of Rotaliidae (mostly Ammonia, particularly A. beccarii var.). Elphidium and Buliminidae are also important in the harbor samples except at Station 3 which is located in the inner part of the harbor.

(2) Percentages of tropical to subtropical species (such as Textularia kerimbaensis, Vertebralina striata, Quinqueloculina parkeri, Miliolinella oceanica, Triloculina berthelotiana, $T$. irregularis, $T$. terquemiana, Spiroloculina corrugata, $S$. foevolata, Articulina fijiensis, Hauerina bradyi, $H$. fragilissima, $H$. pacifica, Peneroplis pertusus, Marginopora hemprichii, Buliminoides williamsoniana, Bolivinella folia, Bolivina rhomboidalis, Sigmavirgulina tortuosa, Loxostomoides mayori, L. durrandii, Trimosina orientalis, Reussella simplex, Pavonina flavelliformis, Hopkinsina pacifica, Glabratella pulvinata makinoi, Angulodiscorbis quadrangularis, Pileolina (?) australensis, P. (?) opercularis, $P$. (?) patelliformis, Planorbulina mediterranensis, $P$. acervalis, Ammonia erinacea, Elphidium craticulatum, Camerina ammonoides, Amphistegina lessonii, Cymbaloporetta bradyi) in the foraminiferal assemblages are very small at Wakaura and Station 3 of Mori Harbor. This is because Wakaura is the northernmost locality among these samples and Stations 3 is located in the inner part of Mori Harbor, and the both localities are not so strongly influenced 
by the warm Kuroshio Current as at the other localities. The highest percentages of tropical species at Seto and Ezura ( $c a .90 \%)$ may be due to the fact that, the places are located close to a point called Bansho-no-saki and is directly influenced by the Kuroshio Current. On the contrary, relatively small percentages of tropical Foraminifera at Kushimoto is unexpected, because Kushimoto is located at the southernmost part of Honshu (main land of Japan). However, its low percentage may be due to the fact that the beach near Kushimoto Railway Station is protected from direct influence of the Kuroshio Current by Shio-no-misaki Peninsula (actually a barrier island) to the west and south, and by Oshima Island to the east.

(3) Among the four samples in and near Mori Harbor, Station 3 is located in the inner part of the harbor, its depth is the shallowest, its sand content is the smallest and, thus, its foraminiferal occurrence is different from that of the other stations. Population of benthonic Foraminifera at Station 3 is smaller than that at the other stations. Foraminifera assemblage at Station 3 is characterized by more abundance of arenaceous Foraminifera, particularly Trochammina cf. globigeriniformis which is characteristic of brackish waters in Japan (UcHro, 1962), more abundance of Ammonia beccarii var., much less occurrence of Elphidium and Buliminidae, and by much less content of tropical to subtropical species. Miliolidae, Elphidium and Buliminidae increase in abundance from Station 3 towards offshore, and Ammonia bececarii var. decreases from Station 3 towards offshore. Thus, Foraminifera distribution of Mori Harbor can be divided into two, inner and outer harbor, assemblages. In connection with the distribution of benthonic Foraminifera in Mori Harbor, YAmazı's plankton study of Tanabe Bay $(1958,1959)$ is of interest. He made a quantitative analysis of the plankton of Tanabe Bay collected in November, 1956 and August, 1957. According to his results, "The distribution of plankton was found to be closely correlated with the hydrographical conditions... The stagnant inner water favors the development of dense zooand phyto-plankton communities which are composed chiefly of smaller organisms as compared with occeanic and neritic forms... Some of the inlet-water forms forming dense populations in the inner area and narrow arms are dispersed towards the entrance of the bay along the southern coast, northern shore and central area where they form tongue-like extension... These organisms (oceanic planktons) which occur abundantly outside the bay flow into the central area of the bay extend to the northeastern areas... The influx of the oceanic waters is not so strong as to influence the condition of the baylet waters... The plankton in inlet waters is used as indicator to distinguish water masses whose chemical and physical properties are unknown or too small to be analysed". Thus, Yamazi's results are very similar to the writer's results based upon the benthonic Foraminifera.

(4) It is of interest to note that many species of benthonic Foraminifera of 
Mori Harbor are in common with those of Recent sediments in Aburatsubo Cove, Miura-shi, Kanagawa-ken and also with those of the Pleistocene shell bed at a great cliff facing Tokyo Bay, at Sakurai, Kisarazu-shi, Chiba-ken. The detailed description of them will be made in another paper in the near future.

In the identification of the Foraminifera species listed in Table 1, the writer has compared his specimens with those of Recent sediments from Dry Tortugas (Florida, U.S.A.), Bermuda, Jamaica, Andros Island (Bahama), Hawaii, New Zealand, Australia, Red Sea and England. Most of these samples are a part of materials from which Cushman originally described his species here in concern. The writer has found several new species (listed in Table 1), but number of specimens of some species are not sufficient and the description of them will be given in another paper very soon.

\section{REFERENCES}

Cushman, J. A. 1948. Foraminifera, their classification and economic use (4th edition). 478 p., 55 pls., Cambridge, Harvard University Press.

FusE, S. 1959. A study on the productivity of Tanabe Bay (Part II). III. Oceanographic conditions of Tanabe Bay (2): Stratification and fluctuation of hydrographical conditions on two sectional survey lines. Rec. Oceanogr. Wks. Jap., Special Number 3, pp. 31-45.

FUSE, S., YAMAZI, I. \& HARADA, E. 1958. A study on the productivity of Tanabe Bay (Part I). I. Oceanographic conditions of Tanabe Bay: Results of the survey in the autumn of 1956. Ibid., Special Number 2, pp. 3-9.

Hay ami, S. \& Fukuo, Y. 1959. A study of the productivity of Tanabe Bay (Part II). VI. On the exchange of water and the productivity of a bay with special reference to Tanabe Bay (2). Ibid., Special Number 3, pp. 61-68.

LOEBRICH, A. R., Jr. \& TAPPAN, H. 1961. Suprageneric classification of the Rhizopoda. J. Paleont. vol. 35 , no. 2, pp. 245-330.

MiYADI, D. \& HAyAmI, S. A study on the productivity of Tanabe Bay (Part I). Rec. Oceanogr. Wks. Jap., Special Number 2, pp. 1-2.

Uchto, T. 1962. Influence of the River Shinano on Foraminifera and sediment grain-size distributions. In: Shepard Commemorative Volume: Papers in Marine Geology, MacMillan Publ. Co., New York (in press).

YAMAZI, I. 1958. A study on the productivity of Tanabe Bay (Part I). IV. On some plankton indicating the water exchange in Tanabe Bay. Rec. Oceanogr. Wks. Jap., Special Number 2, pp. 25-35.

1959. A study on the productivity of Tanabe Bay (Part II). II. On some plankton indicating the water exchange in Tanabe Bay in August, 1957. Ibid., Special Number 3, pp. 23-30.

BRADSHAW, J. S. 1957. Laboratory studies on the rate of growth of the foraminifer 'Streblus beccarii (LINNE) var. tepida (CUSHMAN)'. J. Paleont., vol. 31, no. 6, pp. 1138-47.

LANKFORD, R. R. 1959. Distribution and ecology of Foraminifera from east Mississippi Delta margin. Bull. Amer. Assoc. Petrol. Geol., vol. 43, no. 9, pp. 2068-99.

Phleger, F. B 1960. Recent sedimentology, northwest Gulf of Mexico; Retrospect and prospect. In : Recent sediments, nothwestern Gulf of Mexico. Ibid., Special Publication, pp. 365-67. 


\section{Postseript}

After examining more materials at these localities, rare occurrences of the following species are noted:

Shiraki....."Discorbis" australis PARR, Buccella (?) cf. nipponica (HuseJIMA \& Maruhasi), Sigmoidella kagaensis Cushman \& Ozawa, Cassidulina sp. B, and Hyalinea balthica (SCHROETER)

Kushimoto...Siphoninoides glabra (HERON-AlLEN \& EARLAND), Gypsina vesicularis (PARKer \& Jones), and Cibicides kiiensis UchIO, n. sp. (MS.)

Mori Harbor Station 4 -..Rectobolivina virgula (BRADY)

Thus, in addition to the 37 species listed (see page 141), Rectobolivina virgula, Loxostomoides limbatum costulatum, Siphoninoides glabra and Gypsina vesicularis should be included in tropical to subtropical species.

Another interesting thing to be noted is that the species found in Mori Harbor are usually smaller and have thinner tests than the species found at the beach sand. This fact does not mean that the harbor environment is unfitted for the growth of Foraminifera, and this interpretation can be supported by the relatively larger population of Foraminifera in the harbor samples than in the beach sand (see Table 1 or 2). Very large population at Kushimoto is due to special sampling as mentioned elsewhere in this paper. The small, fragile (thin-walled), and yet, abundant Foraminifera of the harbor sediment can be interpreted that they are well fitted to the quiet and muddy bottom of the harbor. In this connection, PhLEger's (1960) suggestion is very interesting: "BRAdShaw (1957) found in experimental cultures of Foraminifera that specimens grown under optimum conditions reproduced rapidly but were always small in size. In contrast, specimens cultured under conditions near their limits of survival grew to large size but did not reproduce. LANKFORD (1959) found that the large populations off the Mississippi Delta, living under presumably optimum conditions, are composed of small specimens. This suggests that the idea of the "depauperate fauna", i.e., one composed of small specimens, may not indicate adverse conditions of the environment but may be the result of a very favorable environment". 